\title{
Stroke in a Young Swimmer
}

\author{
Shahram Mohaghegh ${ }^{1, *} ;$ Maryam Hajian $^{2}$ \\ ${ }^{1}$ Sports and Exercise Medicine Department, Shahid Beheshti University of Medical Sciences, Tehran, IR Iran \\ ${ }^{2}$ Sports Medicine Research Center, Neuroscience Institute, Tehran University of Medical Sciences, Tehran, IR Iran \\ *Corresponding author: Shahram Mohaghegh, Sports and Exercise Medicine Department, Shahid Beheshti University of Medical Sciences, Tehran, IR Iran. Tel: +98-2155419005, Fax: \\ +98-2155418914, E-mail: sh.mohaghegh@sbmu.ac.ir
}

Received: October 1, 2014; Revised: December 20, 2014; Accepted: December 30, 2014

\begin{abstract}
Introduction: Arterial dissections are important causes of stroke in the young population. Dissection has been reported in association with some sports. It seems that this report is among the first ones of the cervical arterial dissection in a young swimmer.

Case Presentation: A 30-year-old male professional swimmer with no history of any major disease suddenly complained of severe ataxia, moderate headache, neck pain, unilateral left facial weakness, and feelings of tingling and paresthesia on the left side of his body and face a few minutes following head and body stretching exercises in land. There was no history of major head or neck trauma, manipulation, and practicing diving skills in the past. Acute infarction of the left cerebellum was diagnosed after performing brain computed tomography and magnetic resonance imaging (with contrast) studies. Cervical magnetic resonance angiography confirmed left vertebral artery dissection as the cause of infarction.

Conclusions: Important differential diagnoses of cervicocephalic arterial dissection include other vascular or neurological causes of head and neck pain and/or local neurological syndromes and other causes of brain ischemia such as cardiac emboli, atherosclerosis, and vasculopathy of brain vessels. It is important that sports medicine practitioners pay attention to this less-diagnosed cause of stroke in young athletes.
\end{abstract}

Keywords: Arterial Occlusive Diseases; Stroke; Swimming

\section{Introduction}

As a cause of stroke, arterial dissections may happen at any age, but they are more common in the young population. Dissections are causes of approximately $20 \%$ of ischemic strokes in young adults. The annual incidence of spontaneous cervical artery dissections has been reported as 2.6 per 100000 in the USA. The mean age of patients was 44 to 46 years in North America and Europe. There is no clear gender or ethnic predominance in patients, but in some studies men were slightly more affected (1, 2). Most dissections occur spontaneously or after minor or trivial injury to the head or neck. Spontaneous cervical artery dissection has been classified as vertebral artery dissection or internal carotid artery dissection (3). Dissection has been reported in association with sports such as tennis (4), volleyball (5), basketball (6), skating (7), diving (8), dancing (9), trampoline use (10), and yoga (11). There are reports of arterial dissection immediately after swimming in a 60-year-old female (12) and also following sea wave trauma to the neck in a 56-year-old man (13). This report may be the first one of the spontaneous cervical arterial dissection in a young swimmer.

\section{Case Presentation}

A 30-year-old male professional swimmer suddenly complained of moderate headache, neck pain, unilateral left facial weakness, and feelings of tingling and paresthesia on the left side of his body and face, a few minutes following head and body stretching exercises in land. There was no history of major head or neck trauma or manipulation. Also, he had not practiced any diving skills in the past. He had severe ataxia and could not walk without help, but he did not have any loss of consciousness. Acute infarction of the left cerebellum was diagnosed after brain computed tomography (CT) and magnetic resonance imaging (MRI) (with contrast) studies. Magnetic resonance angiography (MRA) of the brain was also done, which was normal. There was no history of major disease in his past medical history. Cardiac and rheumatological workups were normal. His brain and neck MRI (with contrast) and cervical MRA can be seen in Figures 1-3.

Copyright ( 2015, Sports Medicine Research Center. This is an open-access article distributed under the terms of the Creative Commons Attribution-NonCommercial 4.0 International License (http://creativecommons.org/licenses/by-nc/4.0/) which permits copy and redistribute the material just in noncommercial usages, provided the original work is properly cited. 
Figure 1. Brain Magnetic Resonance Imaging of the Patient with Contrast

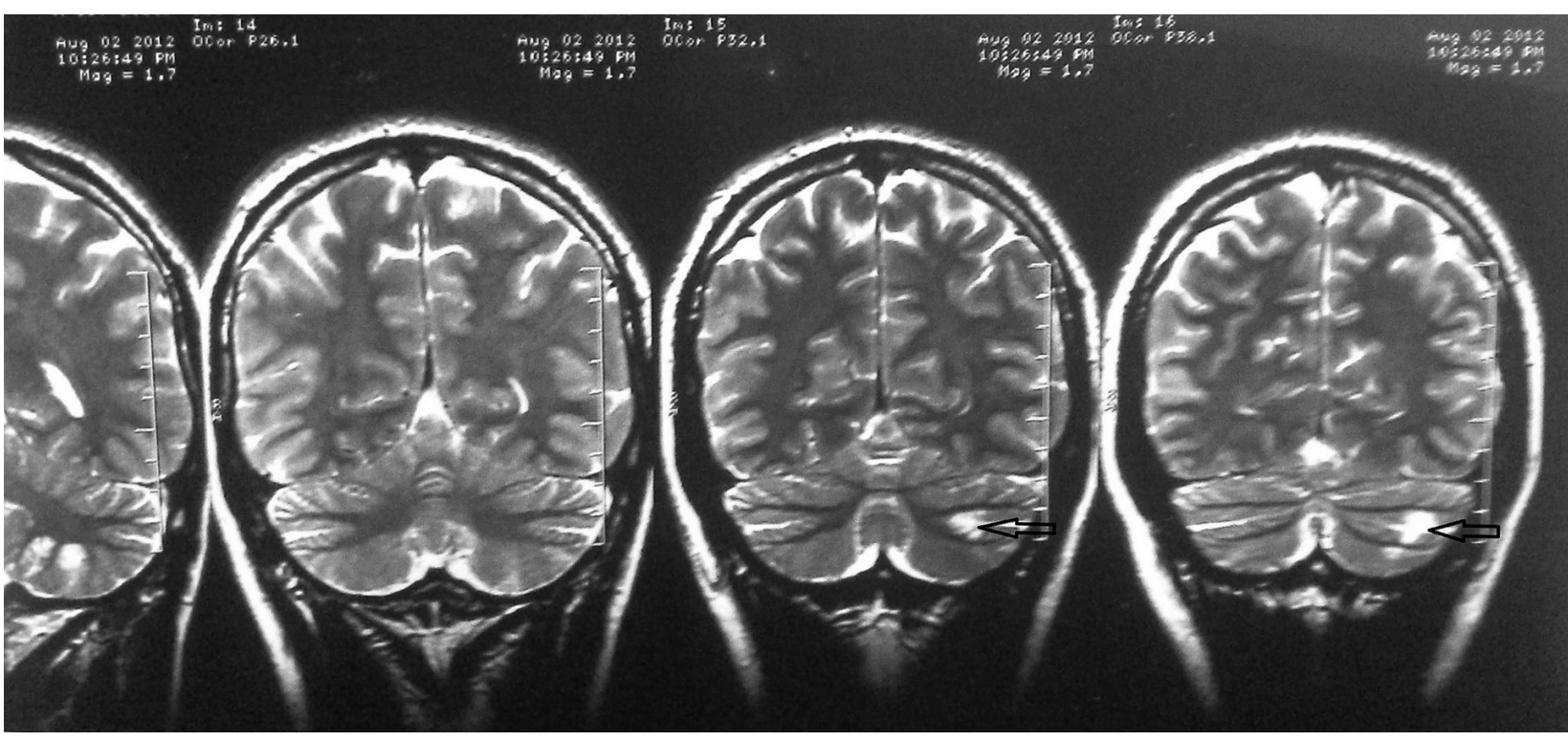

Acute infraction of the left cerebellum is seen (arrows).

Figure 2. Cervical Magnetic Resonance Imaging (MRI) of the Patient with Contrast

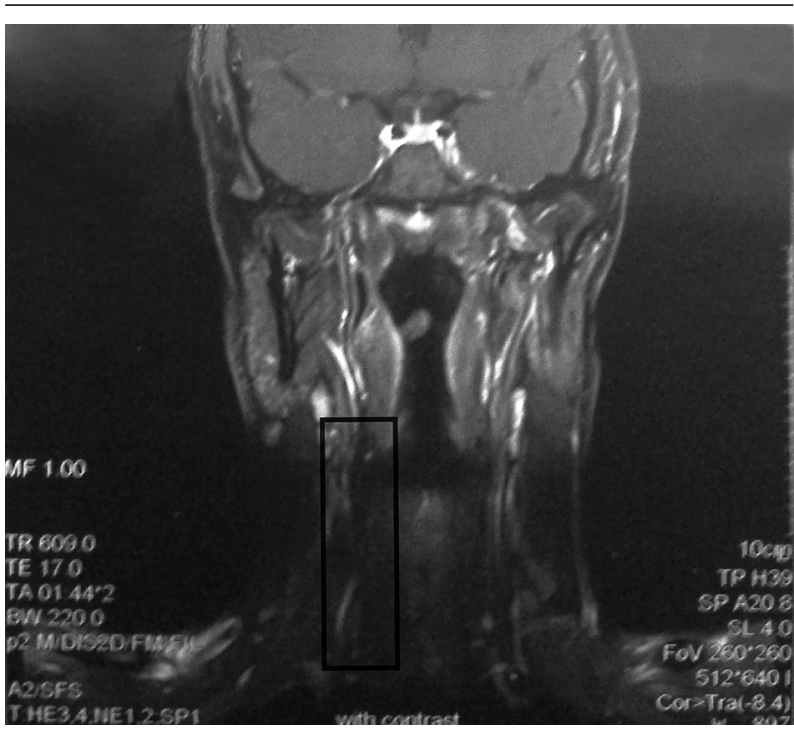

Luminal abnormalities of the left vertebral artery are seen in the box.

\section{Discussion}

Dissection usually causes an ischemic stroke or transient ischemic attack. The development of specific neurological symptoms and signs are related to the ischemia of tissues in the territory of the involved arteries or may result from the compression of the adjacent tissues by the aneurysmal dilatation of the dissected artery.
Figure 3. Cervical Magnetic Resonance Angiography (MRA) of the Patient

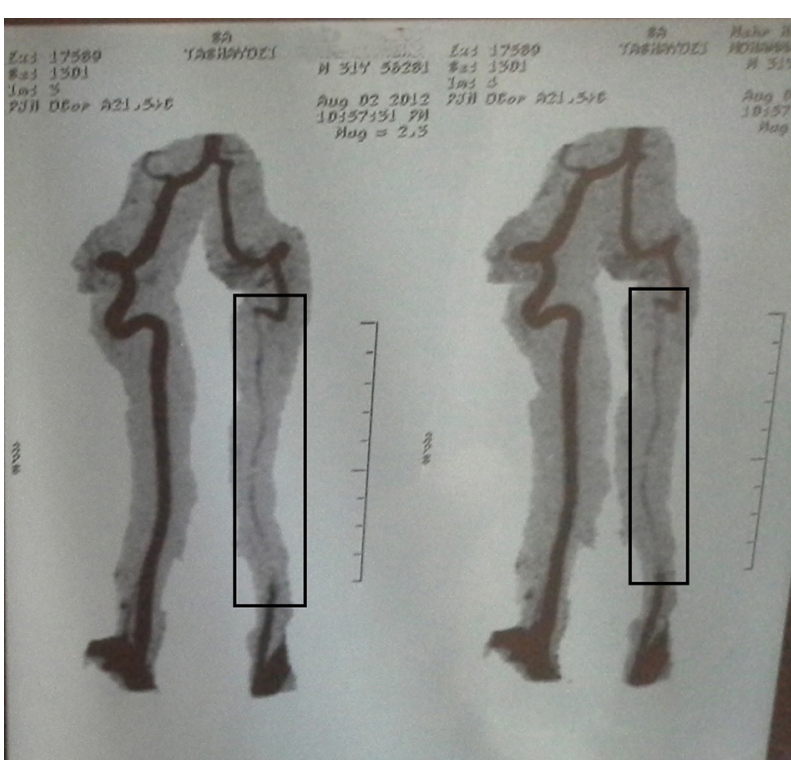

A low flow at the left vertebral artery with a normal flow at the proximal and distal portions can be seen in the boxes.

They include local symptoms and signs such as head and/ or neck pain (the two most frequent initial symptoms), Horner's syndrome (approximately in $25 \%$ of cases), retinal artery occlusion, tinnitus, audible bruit, cranial neuropathies, scalp tenderness, orbital or monocular pain (rare in carotid artery dissections), cervical nerve root 
involvement (rare in vertebral artery dissection), or more generalized presentations such as cerebral ischemia and infarctions and in the case of vertebral artery dissection, lateral medullary infarction (Wallenberg's syndrome) and other posterior circulation territory infarctions and spinal cord ischemia. Subarachnoid hemorrhage rarely occurs ( $1 \%$ of patients). The diagnosis of dissection is usually made with MRI or MRA of the brain or neck. Computed tomography angiography can be used for the diagnosis of cervicocephalic arterial dissection with the same sensitivity and specificity as MRA. Conventional angiography is reserved for younger patients in whom despite high clinical suspicion, there are no conclusive findings in noninvasive imaging studies. Important differential diagnoses of cervicocephalic arterial dissection include other vascular or neurological causes of the head and neck pain and/or local neurological syndromes (such as subarachnoid hemorrhage, migraine, and cluster headache) and other causes of brain ischemia such as cardiac emboli, atherosclerosis, and vasculopathy of brain vessels (14).

In the cervical MRI of this patient (with contrast), there were luminal abnormalities of the left vertebral artery such as arterial wall disruption and intramural hematoma (Figure2).In the neck MRA of this patient, string sign could be observed with a low flow at the left vertebral artery with a normal flow at the proximal and distal parts of the artery, which are diagnostic of dissection (15) (Figure 3).

Also, the diagnosis of cardiac emboli and vasculopathy of the brain vessels was not considered for our patient as he did not have any history of cardiac or rheumatologic diseases and echocardiographic and brain MRI studies were negative for the findings of these diseases. It is important that sports medicine practitioners pay attention to this less-diagnosed cause of stroke in young athletes.

\section{Authors' Contributions}

Both authors have been involved in Concept, gathering information of the case and approval of the article. The corresponding author was responsible of manuscript preparation and critical revision of it.

\section{References}

1. Lee VH, Brown RJ, Mandrekar JN, Mokri B. Incidence and outcome of cervical artery dissection: a population-based study. Neurology. 2006;67(10):1809-12.

2. Debette S, Leys D. Cervical-artery dissections: predisposing factors, diagnosis, and outcome. Lancet Neurol. 2009;8(7):668-78.

3. Hsu YC, Sung SF. Spontaneous vertebral artery dissection with thunderclap headache: a case report and review of the literature. Acta Neurol Taiwan. 2014;23(1):24-8.

4. Abe A, Nishiyama Y, Kamiyama H, Kitahara I, Katsura K, Katayama Y. Symptomatic Middle Cerebral Artery Dissection in a Young Tennis Player. J Nippon Med School. 2009;76(4):209-11.

5. Slankamenac P, Jesic A, Avramov P, Zivanovic Z, Covic S, Till V. Multiple cervical artery dissection in a volleyball player. Arch Neurol. 2010;67(8):1024.

6. Giorgio FD, Vetrugno G, Mercurio DD, Pascali VL, Rainio J, Carbone A, et al. 1. Dissection of the Vertebral Artery During a Basketball Game. Medicine, Science and the Law. 2004;44(1):80-6.

7. Karnik R, Rothmund T, Bonner G, Valentin A, Reuther G. Inline skating as a possible cause of consecutive bilateral vertebral artery dissection. Acta Neurologica Scandinavica. 2000;101(1).

8. Furtner M, Werner P, Felber S, Schmidauer C. Bilateral Carotid Artery Dissection Caused by Springboard Diving. Clin J Sport Med. 2006;16(1):76-8.

9. Faivre A, Abdelfettah Z, Rodriguez S, Nicoli F. Neurological picture. Bilateral internal carotid artery dissection due to elongated styloid processes and shaking dancing. J Neurol Neurosurg Psychiatry. 2009;80(10):1154-5.

10. Wechsler B, Kim H, Hunter J. Trampolines, Children, and Strokes. Am J Physical Med Rehabilitation. 2001;80(8):608-13.

11. Caso V, Paciaroni M, Bogousslavsky J. Environmental Factors and Cervical Artery Dissection. Front Neurol Neurosci. 2005;20:44-53.

12. Kawajiri K, Kiyama M, Hayazaki K. Spontaneous Dissection in the Common Carotid Artery. Neurologia medico-chirurgica. 1995;35(6):373-6.

13. Pego-Reigosa R, Lopez-Lopez S, Vazquez-Lopez ME, Armesto-Perez V, Branas-Fernandez F, Martinez-Vazquez F, et al. Sea wave-induced internal carotid artery dissection. Neurology. 2005;64(11):1980.

14. Leibeskind DS, Saver J. Spontaneous cerebral and cervical artery dissection: Clinical features and diagnosis. 2014 Available from: http:/| www.uptodate.com/contents/spontaneous-cerebral-and-cervicalartery-dissection-clinical-features-and-diagnosis?source=see_link.

15. Gottesman RF, Sharma P, Robinson KA, Arnan M, Tsui M, Saber-Tehrani $\mathrm{A}$, et al. Imaging characteristics of symptomatic vertebral artery dissection: a systematic review. Neurologist. 2012;18(5):255-60. 